\title{
A coupled thermal and electrochemical study of lithium-ion battery cooled by paraffin/porous-graphite-matrix composite
}

\author{
Angelo Greco and Xi Jiang * \\ Department of Engineering, Lancaster University, Lancaster LA1 4YR, UK \\ * Corresponding author. Office phone number: (+44) 1524 592439; E-mail: x.jiang@lancaster.ac.uk
}

\begin{abstract}
Lithium-ion (Li-ion) battery cooling using a phase change material (PCM) / compressed expanded natural graphite (CENG) composite is investigated, for a cylindrical battery cell and for a battery module scale. An electrochemistry model (average model) is coupled to the thermal model, with the addition of a one-dimensional model for the solution and solid diffusion using the nodal network method. The analysis of the temperature distribution of the battery module scale has shown that a twodimensional model is sufficient to describe the transient temperature rise. In consequence, a twodimensional cell-centred finite volume code for unstructured meshes is developed with additions of the electrochemistry and phase change. This two-dimensional thermal model is used to investigate a new and usual battery module configurations cooled by PCM/CENG at different discharge rates. The comparison of both configurations with a constant source term and heat generation based on the electrochemistry model showed the superiority of the new design. In this study, comparisons between the predictions from different analytical and computational tools as well as open-source packages were carried out, and close agreements have been observed.
\end{abstract}

\section{Keywords:}

Battery thermal management, Electrochemistry, Li-ion battery, Phase change material, Porousgraphite-matrix. 


\section{Nomenclature}

$a_{s} \quad$ Active surface area $\left[\mathrm{m}^{2}\right]$

A Geometric area of the electrodes $\left[\mathrm{m}^{2}\right]$

$c_{e} \quad$ Concentration of $\mathrm{Li}^{+}$in the electrolyte [mol.m ${ }^{-3}$ ]

$c_{s} \quad$ Concentration of reduced lithium in the solid phase [mol.m ${ }^{-3}$ ]

$c_{p} \quad$ Specific heat per unit mass $\left[\mathrm{J} \cdot \mathrm{kg}^{-1} \cdot \mathrm{K}^{-1}\right]$

C $\quad$ Capacity of the battery cell [Ah]

$D_{e} \quad$ Electrolyte phase diffusion coefficient $\left[\mathrm{m}^{2} \cdot \mathrm{s}^{-1}\right]$

$D_{s} \quad$ Solid phase diffusion coefficient $\left[\mathrm{m}^{2} . \mathrm{s}^{-1}\right]$

F $\quad$ Faraday constant $\left[\mathrm{C} \cdot \mathrm{mol}^{-1}\right]$

$G_{e} \quad$ Conductance of electrolyte solution $\left[\mathrm{m}^{-3} \cdot \mathrm{s}^{-1}\right]$

$G_{s} \quad$ Conductance of electrode $\left[\mathrm{m}^{-3} \cdot \mathrm{s}^{-1}\right]$

$h_{r} \quad$ Heat transfer coefficient in the radial direction $\left[\mathrm{W} \cdot \mathrm{m}^{-2} \cdot \mathrm{K}^{-1}\right]$

$i_{0} \quad$ Exchange current density [A.m $\left.{ }^{-2}\right]$

I Current intensity flowing through the system [A]

$j_{f} \quad$ Current per unit volume $\left[\right.$ A. $\left.\mathrm{m}^{-3}\right]$

$L \quad$ Length of the solution phase in the electrochemistry model [m]

$L_{b} \quad$ Height of the battery cell [m]

$L_{\text {fin }} \quad$ Length of a fin [m]

$l_{f} \quad$ Latent heat of fusion $\left[\mathrm{J}_{\mathrm{kg}} \mathrm{kg}^{-1}\right]$

$q_{k} \quad$ Production term in the solution phase in the domain $k\left[\mathrm{~mol} . \mathrm{s}^{-1} \cdot \mathrm{m}^{-3}\right]$

$R \quad$ Ideal gas constant $\left[\mathrm{J} \cdot \mathrm{K}^{-1} \cdot \mathrm{mol}^{-1}\right]$ 
$R_{\mathrm{s}} \quad$ Radius of spherical particle in the electrode [m]

$\Delta S \quad$ Entropy of an electrode $\left[\mathrm{J} \cdot \mathrm{K}^{-1} \cdot \mathrm{mol}^{-1}\right]$

$t \quad$ Time $[\mathrm{s}]$

$t^{+} \quad$ Li-ion transference number

$T \quad$ Temperature $\left[{ }^{\circ} \mathrm{C}\right]$

U Thermodynamic equilibrium voltage [V]

$V \quad$ Volume allocated to a node $\left[\mathrm{m}^{3}\right]$

$V_{\text {cell }} \quad$ Cell voltage [V]

$W_{\text {fin }} \quad$ Width of a fin [m]

$x_{s} \quad$ Normalised Li-ion concentrations at the surface of an electrode

Greek letters

$\alpha_{o x} \quad$ Charge transfer coefficient of anodic reaction

$\alpha_{\text {red }} \quad$ Charge transfer coefficient of the reduction reaction

$\delta \quad$ Length of electrode and separator [m]

$\varepsilon_{e} \quad$ Volume fraction of the electrolyte

$\varepsilon_{s} \quad$ Volume fraction of the active material

$\varepsilon_{f} \quad$ Volume fraction of the filler

$\rho \quad$ Density $\left[\mathrm{kg} . \mathrm{m}^{-3}\right]$

$\phi \quad$ Electric potential [V]

$\varphi_{\text {gen }} \quad$ Heat generation [W]

$\gamma \quad$ Flux of particle in the solid phase [mol. s${ }^{-1}$ ]

$\eta \quad$ Electrode overpotential [V]

$\kappa \quad$ Ionic conductivity [S.m $\left.{ }^{-1}\right]$ 
$\sigma \quad$ Solid phase conductivity [S.m ${ }^{-1}$ ]

Subscripts and acronyms

AM Average model

Brugg Bruggman coefficient

CENG Compressed expanded natural graphite

$e \quad$ Electrolyte

$\max \quad$ Maximum

$\min \quad$ Minimum

$n \quad$ Negative electrode

ox Relative to oxidation reaction

$p \quad$ Positive electrode

PCM Phase change material

PP Porous electrode with polynomial approximation

P2D Pseudo two-dimensional

red Relative to reduction reaction

$s \quad$ Solid phase

sep Separator in the electrolyte domain

SOC State of charge

SP Single particle

\section{Superscripts}

eff Effective

surf Relative to the surface of the spherical particle at the electrode 


\section{Introduction}

Electrical and hybrid electrical vehicles are alternatives in the global effort on reducing the carbon dioxide emissions from the transport sector. Batteries are normally used as the power source for these vehicles, and the high power requirement has made the Li-ion battery the best candidate. However, the performance of Li-ion battery is limited by temperature during the charge/discharge processes, despite its high capacity and energy density. Temperature affects the reliability, safety, and efficiency of the battery. Besides, the temperature uniformity is important in order to avoid thermal runaway and hot spots, leading to short circuit in the battery module [1, 2].

The developments of efficient and reliable cooling solutions were investigated and can be classified into two main categories. In active cooling methods, heat is removed by the movement of a coolant in the battery module. A dedicated pump which uses the energy from the battery in order to move the fluid and the cooling flow is adapted in terms of the battery heat generation status, during the driving operation. The systems are usually bulky and require a parallel configuration in order to maintain uniformity between each battery in the module [3, 4]. In addition, studies were performed in order to optimise the flow management system $[5,6]$ by reducing the pump power while keeping the cooling effective.

The other category is dedicated to the passive cooling management of the battery using the phase change phenomenon. Heat pipes based on the liquid/vapour phase change were studied experimentally [7, 8] and theoretically/numerically [9] in battery thermal management. The design of a heat pipe set in the case of prismatic battery cell showed a high level of effectiveness [9]. The solid/liquid phase change phenomenon was used in order to reduce the temperature rise of the battery during discharge. Passive cooling using phase change material showed a good capacity in maintaining the temperature uniformity, despite extreme environmental temperature conditions [10]. However, their low conductivity limited their applications $[11,12]$. In this context, composite materials made of high conductive material and PCM were investigated in order to study their effectiveness in the thermal management of the discharge process.

Kizilel et al. [13] showed that a composite made of a graphite matrix and PCM was able to bring back the battery module to thermal uniformity after a thermal runaway. However, the equations presented did not consider the effect of the latent heat. The experimental properties of the compressed expanded natural graphite (CENG)/PCM composite [14] were used by Greco et al. [15] with the consideration 
of the phase change of the PCM, in order to develop a model predicting the thermal behaviour of a cylindrical battery cell cooled by this composite. This model can be used for designing the composite and to predict the temperature at any points in the battery cell. In addition, design parameters were highlighted which can be optimised for achieving maximum cooling effectiveness.

The main objective of the cooling process is to maintain the temperature under a certain limit. Simplifications such as constant heat generation source in the battery were used in studies carried out previously. Sato [16] studied experimentally the thermodynamics of a Li-ion battery and decomposed the heat generated into a reaction heat, polarisation heat and Joule heat. The Joule and polarisation heating was represented by resistances depending only on the state of charge (SOC). The reaction heat due to the chemical reaction during charge or discharge was taken only depending on the SOC.

Despite the absence of temperature dependency in the heat generation model, the results were close to the experiment but discrepancies appeared after a SOC of 50\%. Nieto et al. [17] simulated the cooling of pouch battery module by cold plates, during charge and discharge from 1C to 3C. The heat generation was represented by the entropy heat and Joule heat, depending on both the SOC and the temperature.

Their model agreed well with the experiment at the lower charge/discharge rate (1C) but unsatisfactorily at the higher rate (3C), with a discrepancy of 7\%. This solution seems satisfactory for representing the heat generation of the battery at low discharge rates. Nonetheless, this model does not give access to electrical parameters of the battery and their behaviour with the rise of temperature. Therefore, an electrochemistry model of the battery cell has to be introduced in order to describe the electrical parameters and to represent a more realistic behaviour of the battery heat generation during charge/discharge.

Based on the porous electrode and concentrated solution theories, the pseudo two-dimensional (P2D) model was developed by the group of Newman $[18,19]$. The model was well validated in galvanostatic discharge (constant discharge rate conditions) and has been widely used. The phase concentration and potential were assumed to vary only in one dimension. The solution domain is taken as continuous and the solid phase is assumed to be composed of identical spherical particles of a pre-determined size in the radial direction. The P2D model can give satisfactory results with appropriate calibrations, but it is time consuming for cycle simulations or when used for on-board battery management system applications. This is why Smith and Wang [20] coupled the P2D model 
with a lumped thermal model in order to explore pulse power limitations and thermal behaviour of a battery pack.

Simplifications were applied to the P2D model and led to the porous electrode model with polynomial approximation (PP) and single particle (SP) models, in order to reduce the computing time. The PP model approximates the solid diffusion with a polynomial function and still takes into account the solution phase diffusion and migration in the electrolyte. The SP model neglects the diffusion in the electrolyte and represents each electrode by a single particle with an area equivalent to the active area of the solid phase described in the P2D model. In addition, the solid diffusion in the SP model is approximated by a parabolic profile. Santhanagopalan et al. [21] compared the three different models at different discharge rates. They observed a close match between the three models at low discharge rates, but larger discrepancy appeared between the P2D (or PP) and SP models at high discharge rates. This difference is mainly due to the absence of solution phase diffusion in the SP model, which has a major impact at high rates.

Moreover, Guo et al. [22] extended the SP model presented by Santhanagopalan et al. [21] with the additions of an energy balance and a solid phase diffusion coefficient depending on the temperature. Their model was validated against the PP model for different galvanostatic operations at low charge/discharge rates. However, the model does not take into account the diffusion in the electrolyte and may lead to discrepancies at high rates.

Similar to the SP model by considering one particle for each electrode, Prada et al. [23] proposed the average model (AM). However, the AM leads to differential equations with the current density approximation, while the SP model gives algebraic-differential equations. Furthermore, the solid and solution phase diffusion was taken into account in the AM, in order to use the model at high discharge rates. The authors coupled the AM with a lumped thermal model made of one node, in order to observe the impact of the temperature on the electrochemistry and also to predict the temperature behaviour of the battery cell during charge/discharge. They linked the internal node of their lumped model to the skin temperature of the battery cell by considering internal and external thermal resistances for modelling the diffusion in the battery cell and the convection cooling with the environment. The comparison with the experiment for low and high charge/discharge rates showed agreements for the cell voltage. The trend of the transient behaviour of the skin temperatures was in good accordance with experiments, but with small discrepancies for the high rates of charge/discharge 
(4C and 8C). The higher temperature prediction observed for the high charge/discharge operations may be associated with neglecting the radiation heat removal and with the consideration of a lumped thermal model with only one node.

Zhang [24] investigated the thermal analysis of a cylindrical Li-ion battery by coupling a SP based model for the electrochemistry with a lumped thermal model taking into account convection and radiation heat removal. The solid diffusion of the Li-ion in the electrode was modelled by a parabolic approximation, and the diffusion of Li-ion in the electrolyte was included in the model. The diffusion in the electrolyte was solved by a finite volume method, where the concentration of lithium was supposed to follow a second-order polynomial function of the coordinate along the thickness direction of the cell components. Moreover, the author showed that the Li-ion concentration gradient in the electrolyte played an important role in the precise determination of the heat generated during charge/discharge operations. The thermal model was simplified by considering only the radial direction with several nodes placed in this direction. The skin temperatures at a discharge rate of 1.7C (5A) predicted by the experiment and the lumped model were $40{ }^{\circ} \mathrm{C}$ and $39.43^{\circ} \mathrm{C}$ respectively, which were in close agreement.

The lumped thermal model is frequently used to model the energy balance when the electrochemistry model is studied with more details. In fact, the coupling of the electrochemistry model with a thermal model remains complex despite the simplifications presented before. The simplification by a lumped model did not take into account the gradient of temperature in the battery cell. This gradient can be important due to the low conductivity of the battery cell in the thickness and radial directions for the prismatic and the cylindrical battery cells respectively. Furthermore, the description of this gradient is important for the design of an efficient battery thermal management system, especially when the design of the cooling system is included in the model.

The one-dimensional (1D) computational model developed by Greco et al. [15] described the temperature behaviour of both battery cell and PCM/CENG with a constant power. However, the constant power assumption poses a severe limitation on its scope of applications. The combined effect of the cylindrical battery cell cooled by a PCM/CENG composite with the additions of the melting process and heat generation described by an electrochemistry model has not been investigated. The present study is motivated by the lack of investigation on the thermal behaviour of the battery cell when electrochemistry is considered. This analysis will allow studying the performances of both 
battery cell and PCM/CENG composite in more realistic conditions. The AM [23] was selected as the electrochemistry model due to its simplicity and satisfactory results against experiments at high and low charge/discharge rates. In addition, the solution and solid diffusion of Li-ion of the AM was replaced by two 1D computational models, validated against analytical solutions.

The aforementioned 1D model was developed for the study of one cylindrical battery cell only and could not be used for simulations of more complex configurations. In consequence, an extension from the one-dimensional model to a multi-dimensional model is performed in order to extend the study of the battery cell cooled by PCM/CENG composite to a battery module scale. The extension was made possible by developing a new code based on the cell-centred Lagrangian diffusion (CCLAD) mathematical method developed by Maire and Breil [25, 26]. This method allows the solution of the thermal equations by a finite volume scheme with anisotropic diffusion on unstructured grids. The phase change model [15] and the modified AM model for the electrochemistry are included in this new code, called CCLAD code for the sake of simplicity. The code is used to compare two different module designs with constant power and power based on the electrochemistry model, i.e. the AM. In this paper, the problem and geometry of a cylindrical battery cell cooled by the PCM/CENG are briefly presented first, followed by the presentation of the electrochemistry model with the description of the modifications applied to it. Next, the analysis of the battery module scale is performed. Comparison is made between the usual and new module designs, with results and discussions subsequently presented. Finally, a summary of the research is presented.

\section{Cylindrical battery cell cooled by a PCM/CENG composite}

The battery cell considered in all the simulations is an ideal cylindrical shaped battery cell with an anisotropic thermal conductivity measured experimentally, i.e. a thermal conductivity of $32 \mathrm{~W} \cdot \mathrm{m}^{-1} \cdot \mathrm{K}^{-1}$ in the axial direction and $0.2 \mathrm{~W} \cdot \mathrm{m}^{-1} \cdot \mathrm{K}^{-1}$ in the radial one [27]. It was shown by Greco et al. [15] that the study of this cylindrical cell, considering the cooling system applied, can be simplified into a twodimensional problem (or a one-dimensional problem due to symmetry by revolution), because of this anisotropic thermal conductivity. This result was also confirmed experimentally and numerically by Zhang [24], Onda et al. [28] and Al Hallaj et al. [29]. 
The cylindrical battery cell is cooled by the PCM/CENG composite, with the battery cell completely surrounded by the cylindrical shape composite. The cylindrical battery cell is represented by a onedimensional [15] model due to the anisotropic thermal conductivity [27] and the PCM/CENG composite is modelled by one equation because of the thermal equilibrium between the PCM and graphite (CENG). The thermal equilibrium between the PCM and graphite is assured by the large surface contact during the melting process. In addition, experimental correlations were used to calculate the density and conductivity of the composite with the modelling of the phase change by an equivalent thermal capacity [15].

In our previous studies, an analytical solution based on the separation variable technique and a 1D computational model based on the thermal network method were developed [9]. The analytical solution took into account the specific heat of the PCM/CENG composite only, while the 1D computational method included the phase change process [15]. The different hypotheses made in place of the development of the analytical solution and 1D computational model were validated against a 3D model simulated by the open-source software SYRTHES4.0 (software dedicated to transient thermal simulations in complex solid geometries by EDF http://researchers.edf.com/software/syrthes-44340.html).

Our previous work considered constant power only, which is not the case for real discharge conditions. In this study, the thermal behaviour of the battery cell cooled by a PCM/CENG coupled with an electrochemistry is investigated in order to predict more realistic behaviours of the battery during discharge process and the impact on the cooling effectiveness of the PCM/CENG composite. The extension of the model will allow analysing the performances of the PCM/CENG composite at different discharge rates. The next section is dedicated to the description of the AM and to the modifications applied to it.

\section{Electrochemistry model}

A Li-ion battery with a lithium iron phosphate cathode and graphite anode is considered in this study, as shown in Fig. 1. The chemical reaction for the discharge of the battery is described by the following reactions.

$$
L i C_{6} \stackrel{\text { Discharge }}{\longrightarrow} x_{s, n} L i^{+}+x_{s, n} e^{-}+L i_{1-x_{s, n}} C_{6} \text { at the negative electrode. }
$$


$x_{s, p} \mathrm{Li}^{+}+x_{s, p} e^{-}+\mathrm{Li}_{1-x_{s, p}} \mathrm{FePO}_{4} \stackrel{\text { Discharge }}{\longrightarrow} \mathrm{LiFePO} 4$ at the positive electrode.

The governing equations and descriptions of the mechanisms during discharge process are presented via the P2D model.

Fig. 1. P2D model of the Li-ion battery.

\subsection{Pseudo two-dimensional model (P2D)}

The equations of the P2D model describe the charge conservation and diffusion of species in the solid and electrolyte phases. The conservation of lithium species is represented by:

$$
\begin{aligned}
& \frac{\partial c_{s}}{\partial t}=\frac{1}{r^{2}} \frac{\partial}{\partial r}\left(r^{2} D_{s} \frac{\partial c_{s}}{\partial r}\right), \\
& \frac{\partial \varepsilon_{e} c_{\mathrm{e}}}{\partial t}=\frac{\partial}{\partial z}\left(D_{e}^{e f f} \frac{\partial c_{e}}{\partial z}\right)+\left(1-t_{+}\right) \frac{j_{f}}{F} .
\end{aligned}
$$

The conservation of charge is represented by the following equations:

$$
\begin{aligned}
& \frac{\partial}{\partial z}\left(\sigma^{e f f} \frac{\partial \phi_{s}}{\partial z}\right)=j_{f}, \\
& \frac{\partial}{\partial z}\left(\kappa^{e f f} \frac{\partial \phi_{e}}{\partial z}\right)+\frac{\partial}{\partial z}\left(\kappa_{D}^{e f f} \frac{\partial \ln c_{e}}{\partial z}\right)+j_{f}=0 .
\end{aligned}
$$

The electrode overpotential and electrochemical kinetics are respectively calculated as follows:

$$
\begin{aligned}
& \eta=\phi_{s}-\phi_{e}-U \\
& j_{f}=a_{s} i_{0}\left[\exp \left(\frac{\alpha_{o x} F}{R T} \eta\right)-\exp \left(-\frac{\alpha_{r e d} F}{R T} \eta\right)\right],
\end{aligned}
$$

with the following boundary conditions:

$$
\begin{aligned}
& D_{s} \frac{\partial c_{s}}{\partial r}(r=0)=0 ;-D_{s} \frac{\partial c_{s}}{\partial r}\left(r=R_{s}\right)=\frac{j_{f}}{a_{s} F}, \\
& \frac{\partial c_{e}}{\partial z}(r=0)=\frac{\partial c_{e}}{\partial z}(z=L)=0,
\end{aligned}
$$


$-\sigma^{e f f} \frac{\partial \phi_{s}}{\partial z}(r=0)=-\sigma^{e f f} \frac{\partial \phi_{s}}{\partial z}(z=L)=\frac{I}{A}$

$\frac{\partial \phi_{s}}{\partial z}\left(r=\delta_{n}\right)=\frac{\partial \phi_{s}}{\partial z}\left(z=L-\delta_{p}\right)=0$,

$\frac{\partial \phi_{e}}{\partial z}(r=0)=\frac{\partial \phi_{e}}{\partial z}(z=L)=0$

During the discharge process, the Li-ion is removed from the sperical sites by diffusion at the negative electrode. This diffusion of Li-ions from the centre to the external face, as described by Eq. (1), is caused by the current generated during the dicharge, as described in Eq. (6). The Li-ions removed from the sperical sites at the negative electrode are transfered through the electrolyte by migration and diffusion in order to reach the positive electerode, as described in Eq. (2). The separator avoids exchanges between the anode and cathode but lets the Li-ions travel through, in order to reach the spherical site of the positive electrode. A diffusion process occurs at the surface of the sphere from the surface to the centre. This insertion of Li-ion in the spherical site at the cathode is also descriped by Eq. (1), with a change of sign for the boundary condition shown in Eq. (1a).

The extraction and insertion of Li-ions at the the negative and positive electrodes generate electrons in the solid phase, collected by the metalic collectors at $\mathrm{z}=0$ and $\mathrm{z}=\mathrm{L}$. An electric potential in the electrolyte exists due to the transfer of charge in the electrolyte, as shown in Eq. (4). It is interesting to notice the conservation of the electric charge by adding Eq. (3) and Eq. (4). The coupling of this model with a detailled themal model is time consuming when cycle simulation and/or pack/module level designs are addressed.

\subsection{Average model}

In this study, the average model proposed by Prada et al. [23] which was based on simplifications of the P2D model is chosen in order to reduce the computing time. The model has shown agreements with experiments for low and high charge/discharge rates. The AM is similar to the SP model by neglecting the solid concentration distribution along the electrode and considering the material diffusion inside a representative solid particle. Nevertheless, the AM leads to partial differential equation with the current density approximation while the SP model gives algebraic-differential equations: 


$$
\begin{aligned}
& \int_{0}^{\delta_{n}} j_{f, n}(z) d z=\frac{I}{A}=\bar{j}_{f, n} \delta_{n}, \\
& \int_{0}^{\delta_{p}} j_{f, \mathrm{p}}(z) d z=-\frac{I}{A}=\bar{j}_{f, \mathrm{p}} \delta_{p} .
\end{aligned}
$$

This approximation transforms the equations of the P2D model into simple partial differential equations which are solved subject to the boundary conditions. In consequence, Eq. (3) and Eq. (4) are integrated for the negative electrode, separator and positive electrode regions with the boundary conditions given in Eq. (3a), Eq. (3b), and Eq. (4a). The solution of these equations led to the following expression of the cell voltage [23]:

$$
\begin{gathered}
V_{\text {cell }}\left(x_{s, p}^{\text {surf }}, x_{s, n}^{\text {surf }}, c_{e}(0), c_{e}(\mathrm{~L}), T\right)=U_{p}\left(x_{s, p}^{\text {surf }}\right)-U_{n}\left(x_{s, n}^{\text {surf }}\right)+\frac{2 R T}{F} \ln \left(\frac{\xi_{p}+\sqrt{\xi_{p}^{2}+1}}{\xi_{n}+\sqrt{\xi_{n}^{2}+1}}\right), \\
+\left(1-t^{+}\right) \frac{2 R T}{F} \ln \left(\frac{c_{e}(\mathrm{~L})}{c_{e}(0)}\right)-\frac{I}{2 A}\left(\frac{\delta_{n}}{\kappa_{n}^{\text {eff }}}+2 \frac{\delta_{\text {sep }}}{\kappa_{\text {sep }}^{\text {eff }}}+\frac{\delta_{p}}{\kappa_{p}^{\text {eff }}}\right)
\end{gathered}
$$

with

$$
\begin{aligned}
& \kappa_{n}^{\text {eff }}=\kappa\left(1-\varepsilon_{f, n}-\varepsilon_{s, n}\right)^{\text {Brugg, },} ; \kappa_{\text {sep }}^{\text {eff }}=\kappa\left(1-\varepsilon_{s, \text { sep }}\right)^{\text {Brugg }, \text { sep }} ; \kappa_{p}^{\text {eff }}=\kappa\left(1-\varepsilon_{f, p}-\varepsilon_{s, p}\right)^{\text {Brugg,p }} ; \\
& \xi_{n}=\frac{R_{s, n}}{6 \varepsilon_{s, n} i_{0, n} A \delta_{n}} I ; \xi_{p}=-\frac{R_{s, p}}{6 \varepsilon_{s, p} i_{0, p} A \delta_{p}} I .
\end{aligned}
$$

The parameters $x_{s, p}^{\text {surf }}=c_{s, p}^{\text {surf }} / c_{s, p, \max }$ and $x_{s, n}^{\text {surf }}=c_{s, n}^{\text {surf }} / c_{s, n, \text { max }}$ represent the normalised Li-ion concentrations at the surface of the cathode and the anode respectively. Furthermore, $U_{p}$ and $U_{n}$ designate the equilibrium potential of the $\mathrm{LiFePO} 4$ electrode (cathode) [30] and the graphite (anode) respectively [20], which are experiment based correlations depending on the normalized Li-ion concentrations at the surface of spherical sites. The cell voltage calculation depends on the solid and solution phase diffusion processes with the necessity of providing the concentration of the lithium at the surface of both electrode particles and also the concentration of $\mathrm{Li}$-ion in the electrolyte at $\mathrm{z}=0$ and $\mathrm{z}=\mathrm{L}$.

As presented before, the addition of the solution diffusion in the electrolyte is important in order to describe properly the heat generation at high discharge rate [21, 23, 24]. The heat generation is defined as follows: 
$\varphi_{\text {gen }}=-I\left(\left(V_{\text {cell }}-\left(U_{p}\left(x_{s, p}^{s}\right)-U_{n}\left(x_{s, n}^{s}\right)\right)\right)+\left(\frac{d U_{p}}{d T}\left(x_{s, p}^{s}\right)-\frac{d U_{n}}{d T}\left(x_{s, n}^{s}\right)\right) T\right)$.

The intensity $I$, is positive for the discharge and negative for the charge. The term $d U / d T$ is linked to the entropy by $d U / d T=\Delta S$ / $F$ when only one electron is exchanged. For the sake of simplicity, $d U / d T$ is called entropy term, with $d U_{p} / d T$ and $d U_{n} / d T$ [31] designating the experimental based correlation of the entropy of the cathode and anode respectively, in terms of the normalized Li-ion concentrations at the surface of spherical sites.

Consequently, the calculation of the cell voltage and heat generation implies knowing the concentration of Li-ion at the surface of the electrode particles, and in the electrolyte. The transformation into simple partial differential equations based on the current density approximation, is used to calculate the Li-ion concentrations in the solid and liquid phases. The determinations of these parameters are performed by resolving the solid and solution diffusion with the nodal network method [9]. The data used to perform the electrochemistry simulation are taken from Prada et al. [23], unless they are stated from other sources.

\subsection{Solid phase diffusion}

The nodal network method used to develop the 1D computational thermal model [15] is applied in this case with the lithium concentration representing the nodes, as shown in Fig. 2(a). The conductance of the electrolyte between two nodes is determined by considering the following particle flux between the nodes:

$$
\gamma_{i, i+1}=-4 \pi D_{s} r^{2} \frac{d c_{s}}{d r}
$$

The integration of Eq. (11) with the hypothesis of a constant flux between the nodes $i$ and $i+1$ gives the final expression of the electrolyte conductance:

$$
c_{s, i}-c_{s, i+1}=\frac{1}{4 \pi D_{s}} \frac{r_{i+1}-r_{i}}{r_{i+1} r_{i}} \gamma ; \frac{1}{G_{s, i, i+1}}=\frac{1}{4 \pi D_{s}} \frac{r_{i+1}-r_{i}}{r_{i+1} r_{i}}
$$

Therefore, each node obeys the following equation from the node 1 to $N-1$ : 
$V_{s, i} \frac{d c_{s, i}}{d t}=G_{s, i, i+1}\left(c_{s, i+1}-c_{s, i}\right)+G_{s, i-1, i}\left(c_{s, i-1}-c_{s, i}\right)$

If $N$ designates the last node, i.e. the node linked to the surface of the spherical particle, the equation becomes:

$V_{s, N} \frac{d c_{s, N}}{d t}=G_{s, N, N-1}\left(c_{s, N-1}-c_{s, N}\right)-4 \pi R_{s}^{2} \frac{\bar{j}_{f}}{a_{s} F}$

A global matrix system is finally obtained and solved similar to the 1D computational thermal model [15] with an implicit Euler decomposition to perform the transient simulation.

Fig. 2. (a) Spatial discretization of the spherical particle in the solid phase, and the normalised Li-ion concentration at the surface of the electrode particles for the discharge rate of: (b) 2C; (c) 4C; and (d) 8C.

The consideration of constant volumetric heat generation for the battery cell $[9,15]$ implies that a steady state is reached as function of the cooling solution and battery geometry at the end of the simulation. Nonetheless, the addition of an electrochemical model changes this behaviour by stopping the simulation when the battery voltage reaches a certain cut-off value or a certain concentration at the surface of the electrode. These values are defined by the manufacturer in order to assure better safety and lifespan of the battery. In consequence, the solid phase diffusion plays an important role in the determination of the heat generation (equilibrium voltage, entropy) and the simulation time.

The insertion/extraction process of Li-ions is very complex due to the partial solution regions and difference in capacity restitutions during charge/discharge processes [23]. A shrinking-core model was developed in order to account for the mechanisms with the use of a particle size distribution. However, such an approach is very time consuming and the use of only one equivalent particle per electrode for the AM does not allow its application. Therefore, the radius of the sphere in the solid phase was taken as dependent on the C-rate by Prada et al. [23], in order to account for differences in capacity restitutions after extraction/insertion of the Li-ions. This adjustment of the particle sizes was in good accordance with experimental results. Table 1 presents the particle size radius dependencies on the discharge rate with $\mathrm{C}=2.3 \mathrm{Ah}$.

[Table 1] 
The 1D computational model of the solid diffusion is compared to the analytical solution developed by Subramanian et al. [32], which was obtained for a constant solid diffusion coefficient $D_{s}$. The study is only focused on the discharge operations, and the simulation stopped (the cut-off condition was met) when the normalised Li-ion concentration reaches $\left.x_{s, n}^{\text {surf }}\right|_{0 \%}$ at the surface of the negative particle or $\left.x_{s, p}^{\text {surf }}\right|_{0 \%}$ at the surface of the positive electrode [23].

The comparison between the analytical solution and the particle diffusion model is performed for 2C, 4C and 8C discharge rates. The results presented in Figs. 2(b), 2(c) and 2(d) showed a close match between the models at different discharge rates, supporting the validity of the solid diffusion computational model. In addition, compared to the analytical one, this model is simple to apply and can be implemented with zones based on different solid diffusion coefficient values and/or solid diffusion coefficients depending on the temperature.

The diffusion of the Li-ions in the solution phase is also described and solved with the same method used in the case of the solid diffusion.

\subsection{Solution phase diffusion}

The diffusion of Li-ion in the electrolyte affects directly the cell voltage and heat generation and has an important impact at high discharge rates. The general equation for each node is presented as follows:

$$
\begin{aligned}
& \varepsilon_{e, k} V_{i} \frac{d c_{\mathrm{e}, i}}{d t}=G_{\mathrm{e}, i, i+1}\left(c_{\mathrm{e}, i+1}-c_{\mathrm{e}, i}\right)+G_{\mathrm{e}, i-1, i}\left(c_{\mathrm{e}, i-1}-c_{\mathrm{e}, i}\right)+q_{k} V_{i} \\
& G_{\mathrm{e}, i, i+1}=\frac{D_{e, k}^{e f f} A}{Z_{i+1}-z_{i}} ; q_{k}=\left(1-t_{+}\right) \frac{j_{f, k}}{F} ; D_{e, k}^{e f f}=D_{e} \varepsilon_{e, k}^{\text {brugg }}
\end{aligned}
$$

with the subscript $k$ designating the anode, cathode and separator. A total of 30 nodes were needed to compute the solution phase diffusion, i.e. 10 nodes per zone (separator, negative and positive electrodes). Considering a constant electrolyte diffusion coefficient and an average current density, an analytical solution for the steady state of the solution phase diffusion is obtained as:

$c_{e}(0)-c_{e}(L)=-q_{n} \delta_{n}{ }^{2}\left(\frac{1}{D_{e, s e p}^{e f f}}-\frac{1}{2 D_{e, n}^{e f f}}\right)+q_{p} \delta_{p}{ }^{2}\left(\frac{1}{D_{e, s e p}^{e f f}}-\frac{1}{2 D_{e, p}^{e f f}}\right)-\frac{q_{p} \delta_{p}}{D_{e, \text { sep }}^{e f f}} L$. 
The transient 1D computational electrolyte diffusion model is compared to the analytical solution obtained for the steady state, as shown in Fig. 3(a).

Fig. 3. (a) Comparison between the transient 1D computational solution diffusion model and the steady state analytical solution for different discharge rates. (b) Solution phase concentration at different discharge rates at $\mathrm{t}=25 \mathrm{~s}$ and $\mathrm{t}=100 \mathrm{~s}$.

The agreement between the two models indicates that the solution phase diffusion model and the number of nodes chosen are adequate. The solution concentrations presented in Fig. 3(a) for different discharge rates, are plotted as functions of the thickness of the cell at $t=25 \mathrm{~s}$ and $t=100 \mathrm{~s}$, in Fig. 3(b). The 1D computational solution of the diffusion has shown its capabilities for describing the transient variation of the Li-ion concentrations in the electrolyte domain.

Furthermore, the solution diffusion model can be implemented with an electrolyte diffusion coefficient depending on the concentration of lithium in the electrolyte and/or the temperature, while the analytical solution describes only the steady state with a constant electrolyte diffusion coefficient for each zone (anode, separator and cathode).

The AM proposed by Prada et al. [23] is modified by the addition of the 1D computational model for the diffusion in the solid and electrolyte phases. Prada et al. [23] used an average constant ionic conductivity based on experimental results due to a lack of experimental correlation for the LiFePO4 and obtained close results to their experiments. This value is used in this study for all the simulations performed.

\section{Analysis of the battery module scale}

This section is dedicated to the analysis of the battery module comprised of the cylindrical batteries considered in section 2, i.e. batteries with anisotropic thermal conductivity [27]. Five identical LiFePO4 cylindrical batteries of $7 \mathrm{~mm}$ radius and $100 \mathrm{~mm}$ height are inserted in the PCM/CENG block. The properties of the PCM/CENG are the same as those used previously [15], i.e. a graphite density of $\rho_{C E N G}=200 \mathrm{~kg} \cdot \mathrm{m}^{-3}$ and the paraffin heptadecane as the PCM. A convective coefficient of 20 $W \cdot m^{-2} \cdot K^{-1}$ is applied on the external surfaces of the battery module (the surface of the PCM/CENG where the battery cells are embedded) with the ambient temperature fixed at $20^{\circ} \mathrm{C}$. The value of 20 W. $\mathrm{m}^{-2} \mathrm{~K}^{-1}$ used by Greco et al. [9, 15] was a test value for model development, which is also within the 
range of practical relevance. For consistency the value is kept the same in this study, which is a further development of the earlier efforts [15].

Moreover, the surfaces in the height direction (z-direction) are assumed adiabatic [15], while the power generation for each battery is taken as constant at $240 \mathrm{~kW} \cdot \mathrm{m}^{-3}$. It was proved numerically and analytically that despite the natural convection taking place on the axial ends, it was still possible to assume those ends as adiabatic [15] for the conditions considered. This assumption was tested to be valid with a very small temperature difference observed by comparing results obtained from natural convection ( $\left.5 \mathrm{~W} \cdot \mathrm{m}^{-2} \mathrm{~K}^{-1}\right)$ applied at both ends and those obtained from adiabatic boundary conditions. The boundary conditions in the z-direction made it possible to study the half-height of the battery module and the ones applied in the $\mathrm{x}$ and $\mathrm{y}$ - directions made it possible to consider only a quarter of the block.

The simulation is performed using SYRTHES4.0 and post-processed using PARAVIEW via SALOME7.4 (software dedicated to build geometry/mesh and post processing of CFD results by EDF http://researchers.edf.com/software/salome-44339.html) for the three-dimension (3D) and twodimensional (2D) simulations. Fig. 4(a) and Fig. 4(b) present the 3D and 2D simulations respectively, of the battery module with the positions of the battery cells.

The maximum temperatures of the battery cells in both 2D and 3D simulations are presented in Fig. 4(c), where $T_{1, \max 1}$ and $T_{1, \max 2}$ represent the maximum temperature of the battery cell 1 , as shown in Fig. 4(a), located at the bottom ( $z=L_{b} / 2$ ) and top ( $z=L_{b}$ ) of the battery cell respectively. An identical notation is applied for battery cell 2 in the $3 \mathrm{D}$ simulation and only $T_{1, \max }$ and $T_{2 \text {,max }}$ are chosen for designating the maximum temperatures of battery cell 1 and cell 2 in the 2D simulation. A close match is observed between the 3D simulation results at different heights (z-direction) and the 2D simulation results. Moreover, the results show that the PCM/CENG matrix is able to maintain the battery cells of the module at a quasi-identical temperature distribution with a maximum temperature difference less than $2{ }^{\circ} \mathrm{C}$ and a minimum skin temperature difference less than $3{ }^{\circ} \mathrm{C}$, between the two cells. This analysis has indicated that a 2D simulation is sufficient to study the behaviour of the battery module embedded in a PCM/CENG block.

Fig. 4. Cylindrical battery cells embedded in a PCM/CENG block: (a) 3D simulation; (b) 2D simulation. (c) Comparison between the 2D and 3D results for battery cells 1 and 2. 
In this study, a small temperature gradient is observed between the battery cells in the module, but it is not significant and all the cells remain quasi-identical, under the action of the PCM/CENG. Nevertheless, this behaviour may change with a larger number of battery cells embedded in the PCM/CENG matrix, because only the external surface would be cooled, therefore leading to larger temperature gradients in the module.

In addition, a new design is investigated in order to reduce the volume of the PCM/CENG used and also to enhance the cooling by increasing its surface. The design presented in Fig. 5 has a thickness of $2 \mathrm{~mm}$ and twelve identical fins integrated into the cooling structure. Each fin has a length of $3 \mathrm{~mm}$ and a width of $1 \mathrm{~mm}$, with the final shape of the PCM/CENG matrix supposed to be moulded [14]. It is worth noting that the prescribed minimum and maximum densities of the CENG matrix for such shape have not been analysed/optimised in order to ensure favourable mechanical properties during operations. Despite this technical uncertainty, the new shape is considered and extended to a battery module made of only 4 battery cells. Each battery is surrounded by the PCM/CENG composite with fins and separated from others by thermal insulators in order to ensure the safety of the battery module. This separation is used for avoiding the propagation of a thermal runaway from one battery to another and also to achieve effective cooling of each cell in the z-direction as shown in Fig. 5. This parallel cooling distribution [4] is aimed to maintain an identical temperature distribution in the battery module.

Fig. 5. A new design of the battery module: a quarter of the battery module cooled by the PCM/CENG with fins $\left(L_{f i n}=3 \mathrm{~mm} ; r_{P C M}=9 \mathrm{~mm} ; W_{f i n}=1 \mathrm{~mm}\right)$.

Since all the battery cells are subject to the same conditions with identical cooling, only one cylindrical battery cell in the new design, i.e. cooled by the PCM/CENG with fins, needs to be studied. Moreover, the symmetries allow using the 1D computational code of the battery cell cooled by a PCM/CENG [15] to study the thermal behaviour of this new configuration. In fact, a 1D thermal model considering the radial direction only was shown to be sufficient to describe the temperature distribution due to the cooling methodology and the anisotropic thermal conductivity of the cell [27]. This radial-only 1D thermal description was also validated experimentally by Prada et al. [23], Zhang [24], Onda et al. [28] and Al Hallaj et al. [29]. The fins in the 1D computational model [15] are only modelled in terms of cooling surface due to the high conductivity of the composite and the small volume occupied by the fins with regard to the rest of the PCM/CENG structure. 
The 1D computational model of the battery cell cooled by a PCM/CENG becomes inadequate for complex geometries and cannot be applied for module or pack studies. The extension of the study to more complex geometries (module/pack designs) with the additions of the phase change and the electrochemistry model has led to develop a new code based on the CCLAD mathematical method. This method originally developed by Maire and Breil $[25,26]$ is a finite volume cell-centred scheme for anisotropic diffusion on unstructured grids.

Considering that a 2D simulation is sufficient to study the module design, the code is developed for 2D geometries and unstructured grids. This code will be designated as CCLAD code in the rest of the article and is used to verify the hypothesis concerning the representation of the fins only in terms of equivalent convection cooling and also, to investigate and compare two different designs of battery module cooling. These two designs are designated by the usual and new designs, where the cylindrical battery cells in the usual design are inserted in a PCM/CENG composite as presented in Fig. 4(b), and the new design is presented in Fig. 5, i.e. the configuration of PCM/CENG with fins.

\section{Comparison between the new and usual battery module design configurations}

The CCLAD code is used to study and compare both the new and usual design configurations presented previously in section 4 . The module in the new and usual configurations is made of four batteries of $7 \mathrm{~mm}$ radius and $65 \mathrm{~mm}$ height. The physical properties of the battery [26] are identical to the one presented in section 2. The volume of the PCM/CENG composite is taken as the same in order to compare the two configurations, where the cooling surface in the new design is 3.7 times of the one in the usual design configuration (Table 2). Due to the symmetries in the $\mathrm{x}$ and y-directions, only a quarter of the module is considered for both the new and usual design configurations.

\section{[Table 2]}

The PCM/CENG composite is identical to the one used before and a convective coefficient of 20 $W \cdot m^{-2} \cdot K^{-1}$ is applied on the cooling surfaces of the module with the ambient temperature fixed at $20^{\circ} \mathrm{C}$. The meshes of the usual and new designs are presented in Fig. 6(a), Fig. 6(b) and Fig. 6(c) respectively, with the PCM/CENG composite coloured in green and the cylindrical battery cell in orange. Both design configurations are studied with a constant power of $240 \mathrm{~kW} \cdot \mathrm{m}^{-3}$ and the electrochemistry model, i.e. the AM. 
Fig. 6. Battery module configurations (a quarter of the domain is shown): (a) the usual design; (b) the new design; and (c) the new design without the fins.

\subsection{Analysis of both configurations with constant heat generation}

The cylindrical battery cell used in both the usual and new designs is the same as the one considered in section 2. The configuration [15] (geometry and cooling process) of the new design allows using the 1D computational model, since all the battery cells of the module are subject to the same conditions. It was considered that the fins of the PCM/CENG composite could be modelled only in terms of convective effect due to the small volume and high conductivity of the composite. The CCLAD code is used in this case to verify this hypothesis. Besides, a comparison with SYRTHES is performed for both the new and usual designs in order to firstly show the effect of the phase change and finally to verify the temperature at the steady state. In fact, only the specific heat of the PCM/CENG composite is implemented into the open-source code SYRTHES, i.e. $l_{f}=0$. Both the CCLAD code and $1 \mathrm{D}$ computational model are expected to reach the same temperature as that obtained by SYRTHES.

Fig. 7(a) shows the comparison of the results. The 1D computational model is not valid to represent the complete transient thermal behaviour due to the simplification made for the description of the fins. Neglecting the volume of the fins leads to a faster temperature rise compared to the case when it is modelled by the CCLAD code. Nonetheless, the steady state is identical for all the models/code, and is confirmed by the comparison with SYRTHES. The usual design configuration is compared to SYRTHES in Fig. 7(b) with a close agreement for the steady state.

The temperature rises for both the new and usual designs are presented in Fig. 7(c) for a constant heat generation. The new design of the PCM/CENG leads to maximum and minimum temperatures of $49.11^{\circ} \mathrm{C}$ and $34.26^{\circ} \mathrm{C}$, while the usual design points to $89.11^{\circ} \mathrm{C}$ and $73.57^{\circ} \mathrm{C}$ respectively.

Fig. 7. (a) Comparison between SYRTHES, CCLAD code and the 1D computational model for the new design with constant heat generation. (b) Comparison between SYRTHES and CCLAD code for the usual design with constant heat generation. (c) Comparison of the maximum and minimum temperatures with constant heat generation between the new and usual designs. 
The comparison between the new and usual design configurations, with a constant heat generation, has shown the superiority of the new design despite an identical volume of PCM/CENG in both configurations. The larger cooling surface obtained by the separation of the cells in the new configuration and the addition of the fins, provide a lower temperature in the module. Although the additions of the fins allow decreasing the maximum temperature, the temperature gradient in a battery cell is not reduced due to the low conductivity of the battery cell in the radial direction. The thermal behaviour of both designs is analysed with the addition of the electrochemistry model subsequently. Moreover, the 1D computational model will only be used to highlight the impact of the fins in the new design configuration.

\subsection{Analysis of both configurations with the electrochemistry model}

The CCLAD code is used to compare the two designs i.e. the new and usual designs, using the same electrochemistry model and simulation conditions in both cases, i.e. same PCM/CENG configuration and convective coefficient. The 4C and 8C discharge rates are investigated and the positions of the cell monitored for this study are presented in Fig. 8(a).

The comparison between the two configurations at different points presents no major differences in terms of temperature as shown in Fig. 8(b). This behaviour is due to the short simulation time, caused by the cut-off conditions and initial capacity of the cell, in which the cooling effects of the fins do not have time to be differentiated from the usual cooling configuration. Besides, it can be seen that a similar behaviour is observed in Fig. 7(c), for the simulation with constant power, where the two design configurations present no difference until $600 \mathrm{~s}$.

In general, a difference can be clearly seen on a battery with a higher heat generation and longer discharge period. Nevertheless, the $8 \mathrm{C}$ discharge rate has a shorter simulation time than the $4 \mathrm{C}$ presented before. Therefore, the initial concentration of Li-ion is artificially increased

$\left(c_{s, n, \max }=60 \cdot 10^{3} \mathrm{~mol} \cdot \mathrm{m}^{-3}\right.$ and $\left.c_{s, p, \max }=40 \cdot 10^{3} \mathrm{~mol} \cdot \mathrm{m}^{-3}\right)$, compared to the ones [23] used in the previous simulations, in order to have a longer simulation time so that the differences between the two design configurations can be observed. This modification implies an increase of the battery capacity, but the discharge is performed at 8C with $\mathrm{C}$ still designating the precious capacity of 2.3Ah [23]. However, the increase of the capacity will imply the increase of the battery size too. Since both the 
new and usual designs are analysed under the same electrochemistry and operating conditions, the new design has been proven to be more effective.

Fig. 8(c) presents the results obtained from the increase of the initial Li-ion concentration in the solid phase. As expected, no difference would have been seen if the original Li-ion concentration values were kept [23], with a cut-off condition at around $200 \mathrm{~s}$ as shown in Fig. 8(c) by the black vertical broken line. Furthermore, the simulation is long enough in order to observe the difference between the two design configurations, with slower temperature rise and lower temperature in the new design compared to the usual one. The results call for efforts on simulating and testing experimentally more powerful cell with longer discharge time.

The last simulation is dedicated to the impact of the fins on the temperature rise, with the simulation setup identical to the previous one for the $8 \mathrm{C}$ discharge rate. In this pursuance, the fins added on the new design are removed, as presented in Fig. 6(c), with the thickness of the PCM/CENG composite kept at $2 \mathrm{~mm}$. Fig. 8(d) shows that the design incorporating the fins leads to maximum and minimum temperatures of $67.55^{\circ} \mathrm{C}$ and $43.52^{\circ} \mathrm{C}$, while the one without them leads to $75.57^{\circ} \mathrm{C}$ and $56.73{ }^{\circ} \mathrm{C}$ respectively. According to this comparison, the impact of the fins is non-negligible on the decrease of the temperature. In addition, the 1D computational model and the CCLAD code are predicting identical results when the geometry does not have the fins.

Fig. 8. (a) Positions and names of the monitored cells. Comparisons between the new and usual designs at T65, T226, T382, T580, T786, T929, T623 for the discharge rate of: (b) the 4C; (c) the 8C, with modified $c_{s, n, \max }$ and $\mathrm{c}_{s, p, \max }$. (d) Comparison of the maximum and minimum temperatures for the new design configuration with and without the fins.

The analysis of the cooling impact of both the new and usual designs on the temperature rise for constant heat generation and an electrochemistry model were performed in this section. The analysis with a constant heat generation showed the superiority of the new module design configuration compared to the usual one. However, the results are different when the electrochemistry is included in the simulation. The short simulation time at high discharge rates $(4 \mathrm{C}, 8 \mathrm{C})$ led to no important differences between the two configurations. In consequence, the initial concentrations of Li-ion were increased for the 8C discharge rate in order to perform a longer simulation, where the differences 
were clearly seen, with advantageous cooling performances for the new design with the fins. In consequence, the usual design is sufficient to cool the batteries, if the battery module is discharged at a high rate during a very short period of time, i.e. when there is no time for the temperature to be differentiated from the new design module configuration. Besides, the addition of the fins implies extra cost due to the additional manufacture procedure and structural constraints.

\section{Summary}

The prediction of thermal and electric properties of the battery during charge/discharge is very important in order to design an efficient battery thermal management system The extension of the cylindrical battery cell cooled by a PCM/CENG composite to a battery module scale was investigated in this study, based on a 1D computational model of a cylindrical battery cell cooled by a PCM/CENG composite [15] in order to predict the temperature behaviour. The heat generated can be approximated with a reversible and irreversible heat source, based on experimental internal electric resistance and experimental entropy data, depending or not, on the temperature and the SOC [16, 17]. This modelling approach is simple and not time consuming, but limited at high discharge without giving detailed electrical information on the battery. An electrochemistry model has been included in the 1D computational model, where the P2D model is used for describing the electrochemistry of the battery cell.

The complexity of the P2D model does not allow its effective use for battery systems equipped with cooling systems, or on board battery management system. In consequence, the SP and PP models were proposed based on simplification of the P2D model and compared [21], with satisfactory results at low discharge rates and discrepancies at high rates for the SP model. The average model proposed by Prada et al. [23] includes the diffusion in the electrolyte and showed satisfactory results compared with experiments at very high discharge rates. In this study, the average model was modified by the addition of the nodal network method [9] to describe the solid and solution diffusion of the Li-ion during charge/discharge operations. The model is simply referred to as the $1 \mathrm{D}$ computational solid diffusion and solution diffusion model. In the case of constant solid diffusion coefficient, it was in good accordance with the analytical solution developed by Subramanian et al. [32]. Close agreement was obtained between the 1D computational solution diffusion model and the steady state analytical solution. 
The AM was included in the 1D computational thermal model [15], but was limited to one cell only. The extension of the model to the module scale was analysed by the comparison between the 3D and 2D simulations of a battery module made of five cells embedded in a PCM/CENG block. These simulations were performed using the open-source software SYRTHES4.0 and showed that a 2D model was sufficient to predict accurately the temperature.

A finite volume code for unstructured meshes, based on the cell-centred Lagrangian diffusion method $[25,26]$, was developed. Since the module scale was shown to be sufficiently described by a 2D problem, the code was only developed for 2D applications using unstructured meshes, with the additions of the phase change and the electrochemistry model.

The CCLAD code was used to investigate a battery module made of four batteries, studied in two different configurations and using the same PCM/CENG volume. The first usual design configuration was composed of the cells embedded in a PCM/CENG block, while the second new design configuration was made of a PCM/CENG including twelve fins in its structure. The comparison between the two configurations with a constant power showed the superiority of the new design. However, the addition of the AM revealed a complete different behaviour. No major discrepancies were observed between the two configurations due to the short simulation time. The simulation time is correlated to the solid diffusion in the electrochemistry model, leading to shorter discharge time at high discharge rate. Accordingly the initial concentration of lithium was increased in order to have a longer simulation. In this case, clear differences were observed between the two configurations and the new design was still superior to the usual one, in terms of cooling performances. Nonetheless, this comparison between the new and usual design has shown that, no major differences may be observed at high discharge rates for a short period. In such a case, the usual design would be preferred due to its simple manufacturing process compared to the new design. In all the simulations performed, the electrochemical model used entropy and equilibrium potential of the electrodes, based on experimental correlation that can be easily modified in order to represent a specific battery.

To further develop and validate the model, experimental studies will be valuable. A more powerful battery with longer discharge time could be useful in order to have more information on the design. Additionally, the CCLAD code as a first prototype is much slower than the 1D computational model for cell simulations. A parallelization of the CCLAD code and its extension to 3D unstructured meshes will be considered in our future work. Hybridisation of the CCLAD method and the 1D 
computational model will be also investigated in order to build a powerful design tool for the PCM/CENG design. It is also important to notice that the CENG density was fixed in this study at the optimum value obtained previously [15]. This parameter was shown to be very important in the development of an efficient PCM/CENG composite. The development of an automatic method to determine the optimised CENG density value in terms of the convective heat, the electrochemistry model and the geometry will also be explored. Finally, the geometry of the battery cells studied in this work was idealised. At very high discharge rates, a resistance at the connection between the wire and the battery cell can be observed and may have an impact on the temperature distribution [33]. This resistance is sometimes estimated or modelled based on experimental results. The influence of this contact resistance may change our predictions by shifting the locations of the maximum temperature and will be investigated on a single cylindrical battery with the current version of the CCLAD code in forced convection cooling and a PCM/CENG cooling.

\section{References}

[1] L. Lu, X. Han, J. Li, J. Hua, M. Ouyang, J. Power Sources 226 (2013) 272-288.

[2] Z. Rao, S. Wang, Renew. Sustain. Energy Rev. 15 (2011) 4554-4571

[3] G. Karimi, X. Li, Int. J. Energy Res. 37 (2013)13-24.

[4] A. A. Pesaran, 2002. J. Power Sources 110 (2002) 377-382.

[5] A. Jarrett, I.Y. Kim, J. Power Sources 196 (23) (2011) 10359-10368.

[6] A. Jarrett, I.Y. Kim, J. Power Sources 245 (2014) 644-655.

[7] M. S. Wu, K. H. Liu, Y. Y. Wang, C. C. Wan, 2002. J. Power Sources 109 (2002) 160 -166.

[8] Z. Rao, S. Wang, M. Wu, Z. Lin, F. Lin, Energy Convers. Manag. 65 (2013) 92-97.

[9] A. Greco, D. Cao, X. Jiang, H. Yang, J. Power Sources 257 (2014) 344-355.

[10] X. Duan, G. F. Naterer, Heat Mass Transf. 53 (2010) 5176-5182.

[11] Z. H. Rao, S. F. Wang, Y. L. Zhang, J. Energy Inst. 85, 1 (2012) 38-43.

[12] M. Y. Ramandi, I. Dincer, G. F. Naterer, Heat Mass Transf. 47 (2011) 777-788.

[13] R. Kizilel, R. Sabbah, J.R. Selman, S. Al-Hallaj, J. Power Sources 194 (2009) 1105-1112. 
[14] X. Py, R. Olives, S. Mauran, Heat Mass Transf. 44 (2011) 2727-2737.

[15] A. Greco, X. Jiang, D. Cao, J. Power Sources 278 (2015) 50-68.

[16] N. Sato, J. Power Sources 99 (2001) 70-77.

[17] N. Nieto, L. Diaz, J. Gastelurrutia, F. Blanco, J. C. Ramos, J. Power Sources 272 (2014) 291302.

[18] J. Newman, K.E. Thomas-Alyea, Electrochemical Systems, third ed., John Wiley \& Sons Inc., New Jersey, 2004.

[19] M. Doyle, T. Fuller, J. Newman, J. Electrochem. Soc. 140 (1993) 1526-1533.

[20] K. Smith, C. Wang, J. Power Sources 160 (2006) 662-673.

[21] S. Santhanagopalan, Q. Guo, P. Ramadass, R. E. White, J. Power Sources 156 (2006) 620-628.

[22] M. Guo, G. Sikha, R. E. White, J. Electrochem. Soc. 158 (2011) 122-132.

[23] E. Prada, D. Di Domenico, Y. Creff, J. Newman, J. Bernard, V. Sauvant-Moynot, F. Huet, J. Electrochem. Soc. 159 (2012) 1508-1519.

[24] X. Zhang, Electrochem. Acta 56 (2011) 1246-1255.

[25] J. Breil, P.-H. Maire, J. Comput. Phys. 224 (2007) 785-823.

[26] P.-H. Maire, J. Breil, J. Comput. Phys. 231 (2012) 2259-2299.

[27] S.J. Drake, D.A. Wetz, J.K. Ostanek, S.P. Miller, J.M. Heinzel, A. Jain, J. Power Sources 252 (2014) 298-304.

[28] K. Onda, T. Ohshima, M. Nakayama, K. Fukuda, T. Araki, J. Power Sources 158 (2006) 535542.

[29] S. Al Hallaj, H. Maleki, J.S. Hong, J.R. Selman, J. Power Sources 83 (1999) 1-8.

[30] U. S. Kasavajjula, C. Wang, P. E. Arcea, J. Electrochem. Soc. 155 (2008) 866-874.

[31] V. V. Viswanathan, D. Choi, D. Wang, W. Xu, S. Towne, R. E. Williford, J. Zhang, J. Liu, Z. Yang, J. Power Sources 195 (2010) 3720-2729.

[32] V. R. Subramanian, R. E. White, J. Power Sources 96 (2001) 385-395.

[33] M. Xu, Z. Zhang, X. Wang, Li. Jia, L. Yang, J. Power Sources 256 (2014) 233-243. 